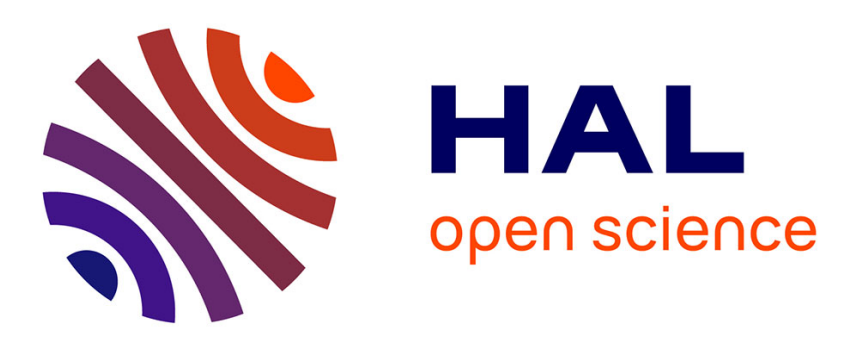

\title{
An overview of Piezoelectric Self-Sensing Actuation for Nanopositioning Applications: Electrical circuits, Displacement and Force estimation
}

Joël Bafumba Liseli, Joel Agnus, Philippe Lutz, Micky Rakotondrabe

\section{- To cite this version:}

Joël Bafumba Liseli, Joel Agnus, Philippe Lutz, Micky Rakotondrabe. An overview of Piezoelectric Self-Sensing Actuation for Nanopositioning Applications: Electrical circuits, Displacement and Force estimation. IEEE Transactions on Instrumentation and Measurement, 2019, 1, pp.1 - 14. 10.1109/TIM.2019.2950760 . hal-02399244

HAL Id: hal-02399244

https://hal.science/hal-02399244

Submitted on 9 Dec 2019

HAL is a multi-disciplinary open access archive for the deposit and dissemination of scientific research documents, whether they are published or not. The documents may come from teaching and research institutions in France or abroad, or from public or private research centers.
L'archive ouverte pluridisciplinaire HAL, est destinée au dépôt et à la diffusion de documents scientifiques de niveau recherche, publiés ou non, émanant des établissements d'enseignement et de recherche français ou étrangers, des laboratoires publics ou privés. 


\title{
An overview of Piezoelectric Self-Sensing Actuation for Nanopositioning Applications: Electrical circuits, Displacement and Force estimation.
}

\author{
Joël BAFUMBA LISELI, Joël AGNUS, Philippe LUTZ , Member, IEEE, and Micky \\ RAKOTONDRABE, Member, IEEE
}

\begin{abstract}
The industrial sector often employs piezoelectric materials as actuators for a variety of uses, some of which requires a precise positioning while being limited by space and cost factors that impede the insertion of external position and force sensors. Piezoelectric Actuators are characterized by strong nonlinearities (hysteresis and creep), badly damped oscillations and sensitivity to the environment, especially temperature variation, that make the measurement of the position mandatory to guarantee the required precision and repeatability of piezoelectricbased positioning systems operating at the micro and nanoscale. Self-Sensing Actuation techniques allow the implementation of precise positioning control of Piezoelectric Actuators without the hindrance of external position sensors. This paper reviews the different Self-Sensing Actuation techniques used for precise positioning control of Piezoelectric Actuators. The principle of Self-Sensing Actuation is defined by the capability of deriving the physical state of a Piezoelectric Actuator (displacement, perceived force, $\cdots$ ) without the use of external sensors to directly measure thereof, but rather by estimating it from the measurement of less intrusive and cheaper physical signals produced by the Piezoelectric Actuator itself (throughout current, voltage drop, $\cdots$ ). The applicability and constraints of each SelfSensing Actuation approach are examined in order to help in the determination of the most adequate approach for precise control of Piezoelectric Actuators positioning and handling force control.
\end{abstract}

Index Terms-Design, Control, Strain, Impedance, Piezoelectric actuators, Sensors, Piezoelectric materials, Force, Automation at micro-nano scales, micro/nano robots, motion control, Force control, Self-Sensing.

\section{INTRODUCTION}

Piezoelectric-based nanopositioning systems generally refer to flexure-hinge-guided mechanisms driven by Piezoelectric Actuators (PEAs). PEAs are electrically controllable positioning element with high precision that have been widely applied in precision types of equipment such as micromanipulators, atomic force microscopes, and ultra-precision machine tools. These kinds of actuators are designed based on the piezoelectric reverse effect. The piezoelectric reverse effect, the key effect used to realise piezo actuator functions, consists in the deformation of a piezoelectric material due to the applied electric field. PEAs play a pivotal role for experimental investigation and manipulation of nanoscale biological, chemical, material, and

The authors are with the Department of Automatic Control and Micro-Mechatronic Systems, FEMTO-ST Institute, Univ Bourgogne Franche-Comté, CNRS, 24 rue Savary, F-25000 Besançon, France. (jbafumba, joel.agnus, philippe.lutz, mratoton) afemto-st.fr physical processes. The high resolution, high bandwidth, small size and high force density of piezoelectric materials make them a good contender as actuators when developing systems acting at the nanoscale.

However, nonlinear effects such as hysteresis and creep affect the position accuracy of piezoelectric-based positioning systems if not compensated [1]. Often, feedback position sensors are mounted to the systems to eliminate the hysteresis and creep [2]-[5]. Nonetheless, the integration of sensors, to enable quality and robust servo control, poses specific problems for microrobots and this is especially true when the number of degrees of freedom (DOF) increases. There are sensors with very good resolutions, sometimes associated with a fairly large range. However, we can note that the best performances on this subject are at the expense of a higher price and a larger footprint (interferometry, encoders). In addition, these sensors mostly only perform measurements with a single DOF, which therefore implies the multiplicity of sensors as soon as a complex measurement is necessary [6]-[9]. Alternative solutions to the integration of external position sensors can be grouped into three categories:

(1) With feedforward voltage control schemes [10], [11] whose performance depends on the accuracy of the PEA's model. Feedforward voltage control schemes do not account for model uncertainties and external disturbances,

(2) With the use of charge amplifiers (charge control scheme), where the PEA is driven by a charge input instead of voltage input [12], [13]. Their performance is related to the hardware complexity. As for feedforward voltage control schemes, charge amplifiers do not account for model uncertainties and external disturbances,

(3) By simultaneously using the piezoelectric material as an actuator and its own sensor, also called Self-Sensing Actuation techniques [14]-[16].

For further performance improvement, combined control schemes such as feedforward-feedback voltage control scheme [17] and integrated voltage-charge control strategy [18] were introduced to overcome limitations imposed by the need to balance a high-bandwidth precision positioning with robust closed-loop stability in the presence of unmodeled dynamics, uncertainties, and disturbances. In the integrated voltagecharge control scheme the charge-based technique handles the hysteresis nonlinearity while the voltage feedforward accounts for the dynamics. In this architecture, the charge 
amplifier is used for a traditional charge based control. This can successfully reduce the hysteresis nonlinearity. However, the charge control can not guarantee the position accuracy in the presence of higher dynamics or external disturbances. The integrated voltage-charge control strategy allows a control of a linearized PEA but lacks robustness against external disturbances and is likely to induce a loss in accuracy during the positioning. Feedforward-feedback voltage control scheme, if properly designed, can be robust to both internal uncertainties in the modeling and external disturbances; however, the feedback branch requires sensors. As already mentioned, unfortunately, accurate sensors are bulky, making them difficult to use for downscaling manipulation systems.

Soft-sensors aid in solving the problem created by the unavailability of embeddable and precise sensors by providing a software backup for it. They are inferential estimators that established themselves as a valuable alternative to the traditional means for the acquisition of process observations when hardware sensors are unavailable or unsuitable. They are based on control theory and provide an estimate of the internal state of a given real system, from available measurements thereof. Software sensors use more suitable hardware sensors that do not directly measure the physical signal of interest; however, associated with an algorithm, they make it possible to reconstruct the signal of interest. Self-Sensing Actuation (SSA) is the PEA's soft-sensor implementation. As for Softsensors, SSA methods do not intend to completely get rid of the hardware sensors but rather to use less bulky and inexpensive sensors associated to an observer/estimator to reconstruct the signal of interest with at least the same resolution as the substituted sensor. The surrogate sensor measures physical signals produced by the PEA itself (throughout current, voltage drop, $\cdots$ ) and allows, thanks to the observer/estimator, to derive its displacement, its perceived strength, or even the temperature variation of its surroundings.

Piezoelectric SSA allows actuating a piezoelectric device while measuring its strain and/or the stress applied uppon it by using itself as its proper sensor. In a piezoelectric SSA the sensor and actuator are truly collocated. Thus, this intrinsic technique may be used in a closed-loop and benefice from a number of advantages related to the closed loop stability known to collocated control [19], [20]. The piezoelectric SSA technique was first proposed in [21] for structures vibration suppression. Sooner, several independent applications began to emerge from vibration suppression of beams [22], [23], scanning tubes [24] to control applications for precise positioning [25], [26]. The main advantage of SSA is the reduction of space occupancy, allowing for better miniaturization of micromanipulation/ microassembly cells. An additional advantage is that the dynamics of the PEA is not affected because no sensor is mechanically attached thereto.

SSA can be classified into two approaches:

(1) SSA based on the piezoelectric direct effect,

(2) SSA based on the PEA's change of electrical properties.

This paper, which reviews the SSA techniques used for both precise positioning and handling force in piezoelectricbased nanopositioning systems, is organized as follows. We begin by reviewing the SSA based on the piezoelectric direct effect techniques, i.e. SSA techniques that employ the PEA's strain-induced charges as a means to estimate its generated displacement. The electrical circuits and constraints on the physical properties of the PEA to be taken into account for its implementation are presented in this section. This is followed by an overview of the SSA based on the PEA's change of electrical properties techniques in Section III. Methods used for the real-time evaluation of the PEA's impedance variation are outlined, accompanied by simplified block diagrams in order to highlight the differences, advantages, and disadvantages to be known before adopting one of these two SSA approaches. Then, a review of emerging solutions for the simultaneous displacement and force estimation from either charge or impedance measurement is provided in Section IV.

\section{SSA BASED ON THE PIEZOELECTRIC DIRECT EFFECT}

A key characteristic of piezoelectric materials is the use of the piezoelectric reverse effect to actuate structures in addition to the piezoelectric direct effect to sense structural deformations. An external force $F_{\text {ext }}$ applied to a piezoelectric material will provoke its deformation $\delta$ and the deformation will cause the apparition of electrical charges $Q_{\delta}$ on the material's surface, this defines the piezoelectric direct effect. A voltage $v_{c}$ applied to the piezoelectric material will generate an electric field $E$ responsible for the transduced force $F_{t}$ that will provoke the deformation $\delta$ of the material, this defines the piezoelectric reverse effect. The piezoelectric reverse and direct effects are not exclusive. The electrodes used to supply the piezoelectric actuators can also be used to get the appearing charges $Q_{\delta}$ that result from its deformation $\delta$. Figure 1 presents an early representation of the electromechanical behavior of piezoelectric transducers.

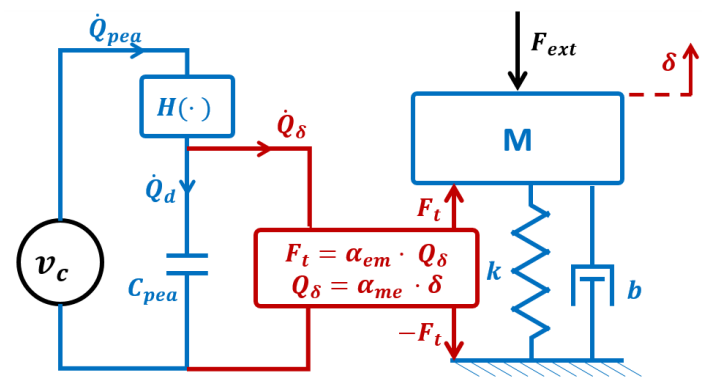

Figure 1: A physical model of the electromechanical behavior of PEAs inspired by Goldfarb et al [27]. This model comprises an electrical domain made of the piezoelectric capacitance $C_{\text {pea }}$ and the operator which describes the hysteresis nonlineraity $\mathrm{H}(\cdot)$. A mass $(M)$ spring $(k)$ damper $(b)$ system to depict the mechanical domain. The transduction factor from the mechanical to the electrical domain $\alpha_{m e}$ and from the electrical to the mechanical domain $\alpha_{e m}$.

When examining PEA physical models [27]-[31] it is noted that two forces can be the causes of the PEA's deformation. These forces are: an external mechanical force $F_{\text {ext }}$ and a transduced force $F_{t}$ due to the application of $v_{c}$. Therefore, whether it is $F_{\text {ext }}$ or $v_{c}$, we observe a deformation $\delta$ of the PEA. According to the piezoelectric direct effect, the deformation of 
a piezoelectric material induces charges $Q_{\delta}$. Furthermore, for a range of frequency, PEAs are known to behaves as capacitors, i.e., the application of an input voltage $v_{c}$ will generate charges $Q_{d}$ due to its dielectric characteristics.

The SSA based on the piezoelectric direct effect aims to separate the charges due to the PEA's deformation $Q_{\delta}$ from those due to the dielectric effect $Q_{d}$ and reconstruct the PEA's displacement $\delta$ from $Q_{\delta}$ without the need of an external position sensor. This is made possible thanks to a dual dielectric feedthrough cancellation that helps recover the signal arising from the PEA's deformation. The dual feedthrough cancellation offsets the PEA dielectric behavior ${ }^{1}$ so that only the signal generated by the PEA's deformation can be measured. This operation is all the more important given that $Q_{d} \gg Q_{\delta}$, i.e., the dielectric signal magnitude is several orders of magnitude higher than that generated by the deformation of the PEA.

For the remainder of this section we will only focus on the electrical domain (see figure 2) for the review of the different dielectric feedthrough cancellation circuits used in the literature. The dielectric feedthrough cancellation circuit to be utilized depends on the adopted strain-electrical signal behavior of the PEA. Two equivalent electrical schematics are used to describe PEAs' behavior:

(1) A capacitor $C_{p e a}$ and a strain-dependent voltage source $v_{\delta}$ in series with $C_{p e a}$ [15], [32]. For static and quasi-static applications, a leaking resistance $R_{L}$ parallel to $C_{p e a}$, commonly negligible but significant at lower frequency to account for current leak, is added to the schematic [33] (see figure 2b).

(2) A strain-dependent charge source $Q_{\delta}$ in parallel with the nominal piezoelectric capacitance $C_{\text {pea }}$ and a leaking resistance $R_{L}$ to account for the leaking current (see figure 2c).

For both schematics, a hysteresis operator $\mathrm{H}(\cdot)$ is used to characterize the hysteresis nonlinearity that typefies PEAs. Nevertheless, for SSA based on the piezoelectric direct effect, the deformation is said to be proportional to the electrical signal it generates. Therefore, although present, the hysteresis is neglected during the determination of the PEA's displacement estimate. One can either use the voltage-based SSA that employs the equivalent voltage source representation or the charge-based SSA that employs the equivalent charge source representation to estimate the strain or perceived force of the PEA.

\section{A. Voltage-based SSA}

As shown in figure $2 \mathrm{~b}$, an additional voltage potential accross the PEA $v_{\delta}$ emerges from its deformation. The voltage $v_{\delta}$ is not detectable directly when a PEA functions as a Self-Sensing actuator because of the control voltage $v_{c}$. To obtain the signal due to the PEA's strain, a capacitance bridge is created similar to that in figure 3 to discriminate $v_{\delta}$ from $v_{c}$. From the diagram

\footnotetext{
${ }^{1}$ For $v_{c}=0$ there is no feedthrough due to the PEA dielectric behavior. Therefore, the PEA behaves as a mere strain sensor.
}

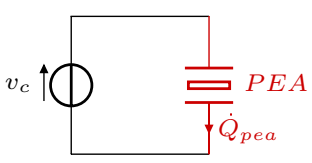

(a) Electrical symbol of a Piezoelectric material.

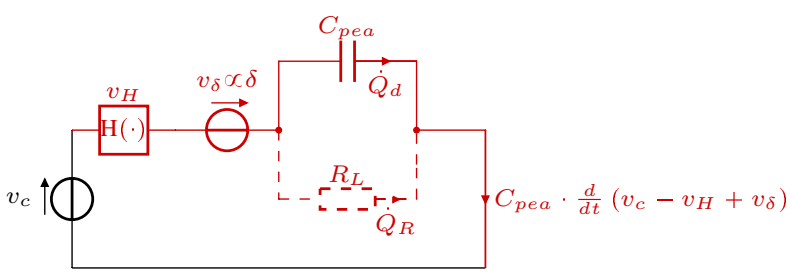

(b) Equivalent voltage source representation of a PEA.

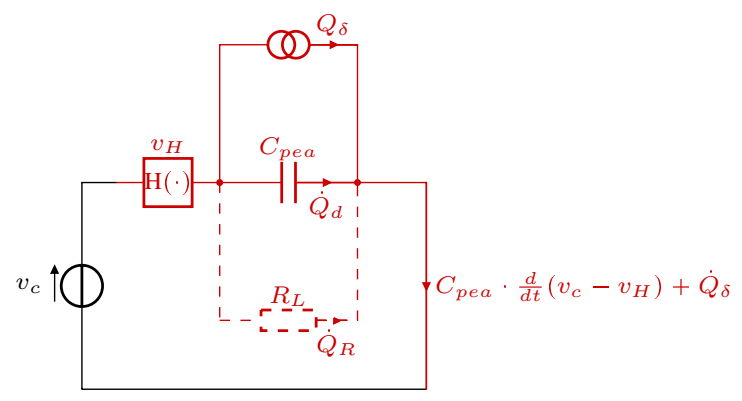

(c) Equivalent charge source representation of a PEA.

Figure 2: Symbol and equivalent electrical models of PEAs. $\mathrm{H}(\cdot)$ expresses the hysteresis nonlinearity and $v_{H}$ the voltage drop due to $\mathrm{H}(\cdot)$. In these figures only the hysteresis nonlinearity is represented because it is the main form of nonlinearity in piezoelectric transducers. The dashed $(---)$ part is to be considered only for lower frequency nearby DC $\left(R_{r}=R_{L}\right)$.

of the strain bridge shown in figure 3 , one can deduce the sensing voltage $v_{s}$ as:

$$
\begin{aligned}
v_{s}= & -\frac{C_{\text {pea }}}{C_{1}+C_{\text {pea }}} \cdot(v_{\delta}-\underbrace{v_{H}}_{\text {neglected }}) \\
& +\left(\frac{C_{\text {pea }}}{C_{1}+C_{\text {pea }}}-\frac{C_{r}}{C_{2}+C_{r}}\right) \cdot v_{c}
\end{aligned}
$$

All the strain bridge's components are chosen so that the bridge circuit is balanced, that is, $C_{p e a} C_{1}=C_{r} C_{2}$ (and $R_{r}=$ $R_{L}$ only for lower frequency nearby DC) so that $v_{s}=0 \forall$ $v_{c}$. The induced voltage $v_{\delta}$ generated due to strain in the PEA creates an imbalance in the bridge. The voltage difference between the two branches provides a sensing signal, $v_{s}=$ $v_{2}-v_{1}$ proportional to the strain induced voltage $v_{\delta}$. The sensing voltage $v_{s}$ of the bridge circuit is only related to the voltage $v_{\delta}$, which is derived from the PEA's strain and is independent of the control voltage $v_{c}$. For $C_{p e a} C_{1}=C_{r} C_{2}$, Eq. 1 can be rewritten as follows:

$$
\begin{aligned}
v_{s} & =-\frac{C_{p e a}}{C_{1}+C_{p e a}} \cdot v_{\delta}+\left(\frac{C_{p e a}}{C_{1}+C_{p e a}}-\frac{C_{r}}{C_{2}+C_{r}}\right) \cdot v_{c} \\
& =-\frac{C_{p e a}}{C_{1}+C_{p e a}} \cdot v_{\delta}
\end{aligned}
$$


Thence, the displacement of the PEA can be estimated through the relationship:

$$
\delta=-\alpha_{v}\left(\frac{C_{1}+C_{p e a}}{C_{p e a}} \cdot v_{s}\right)
$$

where $\alpha_{v}$ is denoted as the PEA voltage-displacement coefficient.

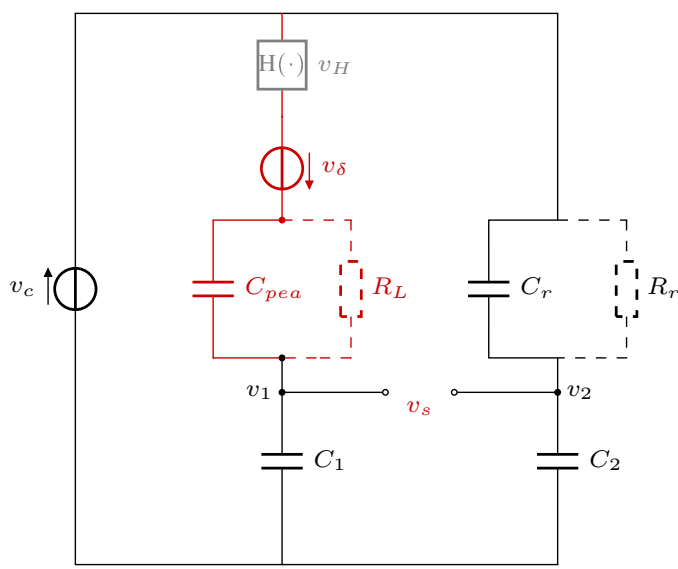

Figure 3: Self-sensing strain bridge for dielectric feedthrough cancellation in case of Voltage-based SSA [33]. For this configuration the hysteresis nonlinearity $\mathrm{H}(\cdot)$ is neglected in the literature. The dashed $(---)$ part is to be considered only for lower frequency nearby $\mathrm{DC}\left(R_{r}=R_{L}\right)$.

Though simple to implement, this technique has several limitations in practice. Here are the most relevant:

- The size of the reference capacitance $C_{r}$ has to be all the time equal to the PEA's capacitance $C_{p e a}$. However, $C_{p e a}$ is greatly influenced by ambient temperature, humidity, preload, and the control input voltage, making the success of voltage-based SSA relies on a tedious continual tuning of the reference capacitance [22], [34]. Since it is difficult to maintain a dynamic balance of the voltage-based SSA, this results in a significant error in signal separation, which hampers the applicability of this technique and adversely affects performance and stability of the closed-loop control system. Ye He et al. [35] proposed an adaptative signal separation algorithm to estimate the gain between halfbridges to automatically adjust the bridge circuit balance to eliminate the part of $v_{s}$ that is related to $v_{c}$. Although innovative, this method does not take into account the impact of the control input voltage, $v_{c}$, on $C_{\text {pea }}$.

- Due to the electromechanical coupling nature of piezoelectric materials, $C_{p e a}$ is not only influenced by ambient temperature, humidity, etc. but even more by its mechanical deformation. Indeed, due to the piezoelectric converse effect, the application of an electric field creates a mechanical deformation in the PEA's crystals and with this deformation, the PEA maintains its volume but does not keep the same geometry due to Poisson effect. This geometry change alters the PEA's electrical properties. Therefore, an equivalent electrical model that does not account for the variations of $C_{\text {pea }}$ due to $v_{c}$ would not accurately depict the electrical characteristics of piezoelectric materials [36].

- The flow of free electric charges responsible for generating $v_{\delta}$ continues until they neutralize the polarization effect. Thus, bridge circuits [21], [32], [37] are often used for strain rate sensing and are effective in suppressing the vibration modes but are not easy to balance for long-term measurements.

- The PEA's strain is said to be linear to the produced charges $Q_{\delta}$. Voltage-based SSA exploits $v_{\delta}$ rather than $Q_{\delta}$. And, $v_{\delta}$ is linked to $Q_{\delta}$ through the relationship ${ }^{2}$ :

$$
v_{\delta}=\frac{Q_{\delta}}{C_{\text {pea }}}
$$

According to Eq. 4 the relationship between $v_{\delta}$ and the PEA's strain depends on $C_{\text {pea }}$ behavior. Since $C_{\text {pea }}$ value varies according to several factors among which $v_{c}$ and $F_{e x t}$, a linear relationship between $v_{\delta}$ and the PEA's generated displacement $\delta$ is not always garanteed.

\section{B. Charge-based SSA}

Charge-based SSA approach relies on the linear chargedeformation relationship of PEAs [38], [39]. It is known that the application of $v_{c}$ on a PEA creates charges due to its dielectric characteristics $Q_{d}$ plus charges due to the PEA's strain $Q_{\delta}$ (see figure 2c). For the linear relationship between the PEA's generated displacement $\delta$ and $Q_{\delta}$ to be exploited, $Q_{\delta}$ must be separated from $Q_{d}$. To this end, Hagood et al. [40] introduced an antiparallel circuit for the offsetting of the dielectric feedthrough $Q_{d}$ to directly measure strain induced charges of the PEA through direct charge amplification (see figure 4). Once this separation is done, $\delta$ can be estimated through the relationship:

$$
\delta=\alpha_{Q} \cdot Q_{\delta}
$$

where $\alpha_{Q}$ is denoted as the PEA charge-displacement coefficient. The coefficients $\alpha_{Q}$ and $\alpha_{v}$ are identified empirically [35], [39].

Like for the voltage-based SSA, only an exact match of the PEA's electrical characteristics in the antiparallel branch allows for perfect discrimination of $Q_{\delta}$ from $Q_{d}$. Therefore, all the above-mentioned drawbacks of the voltage-based SSA are to be considered for the charge-based SSA. Nevertheless, whereas for the Self-Sensing strain bridge, the three capacitors must be taken into account to balance the bridge, in the case of the antiparallel circuit, only the capacitor of the antiparallel branch is taken into account. This makes the creation of an antiparallel branch that accounts for all the PEA's defects (e.g. relaxation time, nonlinearities, $\cdots$ ) feasible. In this case, a thorough analysis of the PEA's electrical characteristics and its dependencies to mechanical and electrical excitations (frequency and amplitude) is required to guarantee the complete offset of $Q_{d}$ and retrievement of $Q_{\delta}$.

Numerous researches have been carried out on the design of equivalent circuit models of piezoelectric materials. Park [41] found that the circuit model with a capacitor and internal

\footnotetext{
${ }^{2} R_{L}$ needs to be considered for lower frequencies nearby DC.
} 


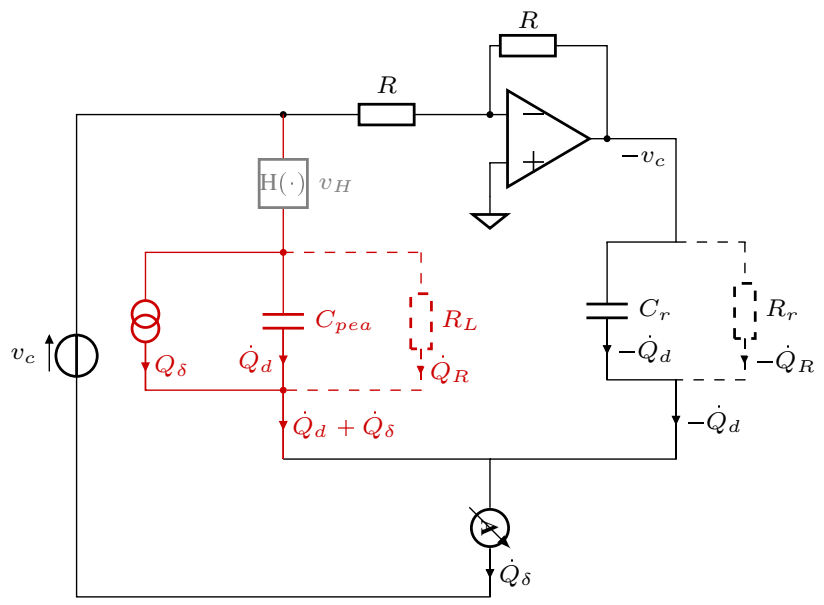

Figure 4: Antiparallel circuit for dielectric feedthrough cancellation in case of charge-based SSA. For this configuration the hysteresis nonlinearity $\mathrm{H}(\cdot)$ is neglected in the literature. The dashed (- - ) part is to be considered only for lower frequency nearby $\mathrm{DC}\left(R_{r}=R_{L}\right)$.

resistor in series could represent the behavior of piezoelectric ceramics much better than the model with capacitor and resistor in parallel when considering the energy dissipation. Mingjie et al [42] combined the behavior of the parallel model at low frequency and that of the series model at high frequency and showed that this representation well fitted with experimental tests. Bafumba et al. [36] captured the influence of the control voltage $v_{c}$ (amplitude and frequency) on PEAs with an impedance map (see figure 5). Mansour et al. [43] has highlighted the effect of an external force on the PEA's impedance. To summarize, to discriminate $Q_{\delta}$ from $Q_{d}$ one needs an electrical component in the antiparallel branch that accounts for humidity, ambient temperature, the control voltage (amplitude and frequency) and the external force applied upon the PEA. Unfortunately, no analog electrical component fits the piezoelectric material impedance dependences. Nevertheless, a numerical equivalent electronic circuit can be designed to integrate all these dependencies for a thorough separation of $Q_{\delta}$ from $Q_{d}$. Considering that a digital impedance is designed for robust discrimination of $Q_{\delta}$ from $Q_{d}$, two problems still need to be addressed:

(1) A piezoelectric actuator or sensor is an electromechanically coupled system, that is, the design for actuation use should be done in consideration of the impact on the sensor aspect and vice versa. Accordingly, one requires a PEA design optimization for both actuation and observation for the applicability of either voltage-based or charge-based SSA.

(2) The transitional behavior of the PEA's strain induced charges to allow for static or quasi-static PEA's displacement estimation. Indeed, after an instantaneous deformation of a PEA due to the application of an electric field, if the electric field is maintained, the PEA will keep deforming due to the creep effect. This deformation, even though small, will keep producing strain-induced
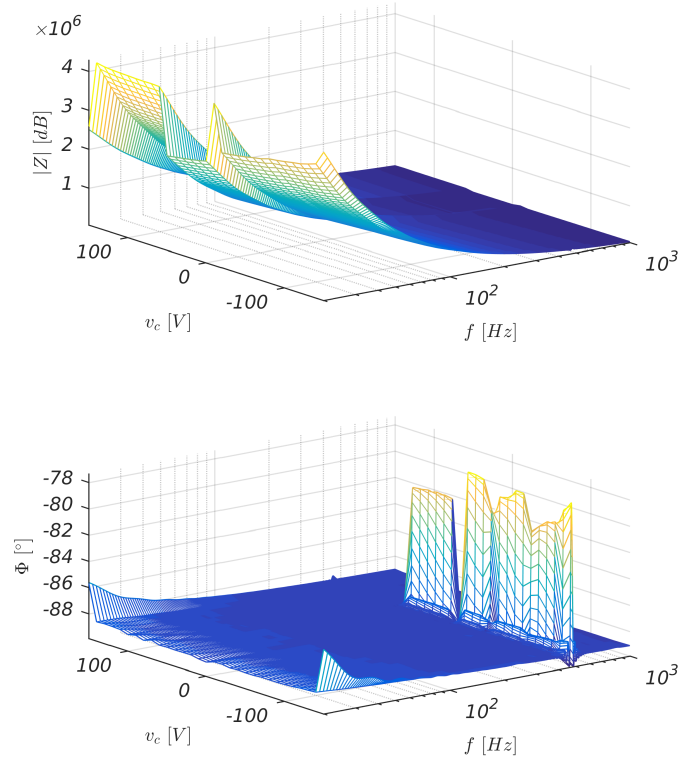

Figure 5: Impact of varying the input voltage amplitude $v_{c}$ and frequency $f$ on the PEA's impedance $|Z|\langle\Phi$ in case of a bilayer unimorph piezoelectric cantilever beam composedd of a $200 \mu m$ PZT upper layer and a $50 \mu m \mathrm{Cu}$ bottom layer

[36]. for $20 \mathrm{~Hz}<f<1 \mathrm{KHz}$, the PEA behaves as a capacitor $\left(\Phi \approx-90^{\circ}\right)$. However, the PEA's impedance magnitude $|Z|$ varies as a result of the PEA's deformation caused by $v_{c}\left(|Z|=h\left(v_{c}\right)\right)$.

charges $Q_{\delta}$, the difference between $C_{p e a}$ and $C_{r}$ will keep increasing and $\alpha_{Q}$ may not longer apply to reconstruct the PEA's displacement $\hat{\delta}$ from $Q_{\delta}$.

Figure 6 presents a simplified block diagram for a simultaneous control and displacement measurement of a PEA using either the voltage or the charge-based SSA. This figure allows apprehending the similarities and subtleties in the implementation of these two SSA schemes based on the piezoelectric direct effect.

\section{Optimal design of piezoelectric-based nanopositioning systems for charge-based SSA}

Even though charge-based SSA has demonstrated a great potential [36], [38], [44], [45], its effectiveness depends on the morphology of the PEA and the choice of the mechanical properties of its constituent materials. Therefore, the first step to take full advantage of the potential of charge-based SSA is the PEA's design.

\section{a) Piezoelectric actuator design optimization:}

The distribution of material inside the piezoelectric layers influences the actuators' performance and hence the number, shape, size, and placement of the actuators have to be optimized. Among the optimization methods for piezoelectric actuators, there are: 


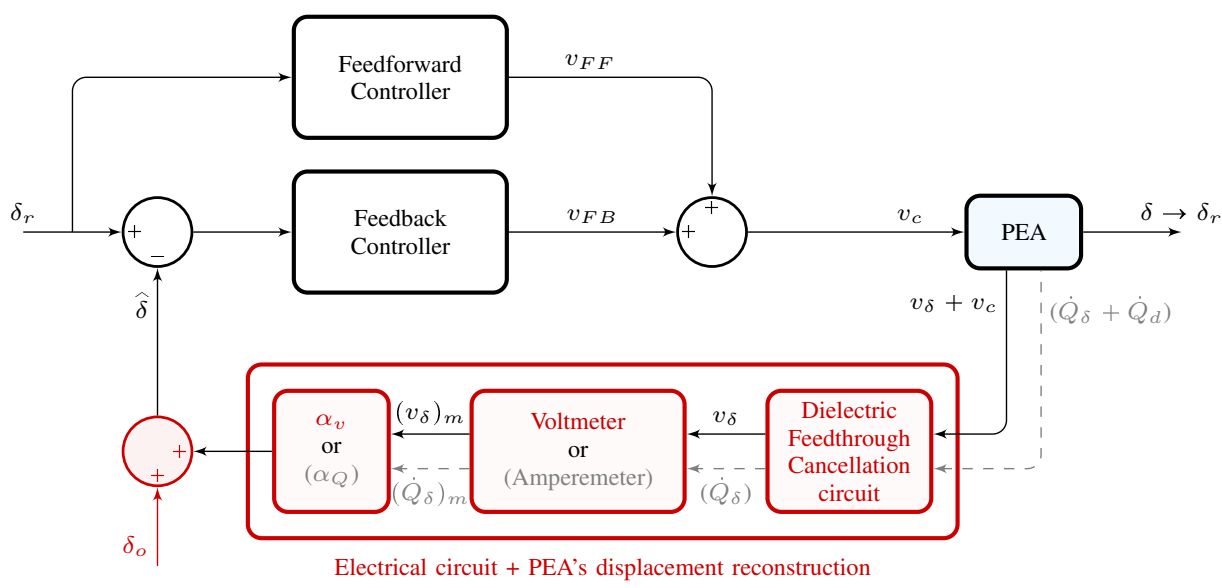

Figure 6: Simplified block diagram of the simultaneous control and displacement measurement of a PEA using Voltage(Charge)based SSA. $\alpha_{v}$ and $\alpha_{Q}$ are The PEA voltage- and charge-displacement coefficient respectively. $\delta_{o}$ is the remaining PEA's displacement due to the previous voltage application and the subscript $(\cdot)_{m}$ stands for measured.

- Parametric optimization in which parameters of the PEA are varyied in order to determine the dimensions and material properties that guarantee improved performances in term of output range [46], [47] $\left(\frac{\delta_{\text {pre }}}{v_{c}}\right)$ and in term of bandwidth [48]. $\delta_{\text {pre }}$ is the bending due to the piezoelectric reverse effect under the application of $v_{c}$.

- Topology optimization which is based on the Piezoelectric Material with Penalization (PEMAP) model, where the design variable is the pseudo-density $\rho_{1}$, which describes the amount of piezoelectric material in each finite element in the piezoelectric layer(s). Topology optimization is employed to find an optimal distribution of piezoelectric material in a multi-layer plate or shell structure to provide the maximum displacement $\delta_{\text {pre }}$ or generated forces in a given direction at a given point of the domain [49]-[51].

- Simultaneous topology and polarization optimization which uses the Piezoelectric Material with Penalization and Polarization (PEMAP-P) and work to find the optimum actuator layout and polarization profile simultaneously [52]-[54]. For this method, in addition to the pseudo-density $\rho_{1}$, a new design variable $\rho_{2}$ is introduced for the polarization of the piezoelectric material. The optimization problem consists in distributing the piezoelectric actuators in such a way as to achieve a maximum output displacement $\delta_{\text {pre }}$ in a given direction at a given point of the structure, while simultaneously minimizing the structural compliance.

All these methods aim to determine the geometric feature that will enhance the PEA's generated displacement due to an applied input voltage without regard to the sensor aspect of the piezoelectric material.

\section{b) Piezoelectric sensor optimization:}

On the one hand, applying an input voltage will result in the piezoelectric material elongation/contraction, and on the other hand, a pressure applied onto a piezoelectric material will be converted into an electrical output (strain-induced charges). When a piezoelectric material is used to convert mechanical into electrical energy it is called a piezoelectric sensor. Like for piezoelectric actuators, works have been conducted for the optimization of piezoelectric sensors.

These searches can be grouped into two categories:

- Geometric optimization methods that aim for the optimization of geometric parameters such as length, width, and thickness of the piezoelectric layer in order to maximize the output recuperated electrical charges at the electrodes $Q_{\delta}$. Pillai et al. [55] presented an analytical method to design an optimal unimorph beam that maximizes the strain-induced charges sensitivity when acted upon by an uniform mechanical load $p$ at a specified frequency $\nu_{s}\left(\frac{Q_{\delta}}{p\left(\nu_{s}\right)}\right)$. Schlinquer et al. [56] suggested an unimorph and Chen et al. [57] a bimorph piezoelectric cantilever mechanical structure optimization for energy harvesting. The optimized design aimed to maximize the PEA's strain-induced charges due to external harmonic load $\left(\frac{Q_{\delta}}{F_{\text {ext }}(\nu)}\right)$, where $\nu$ is the frequency of the harmonic load.

- Localization optimization methods that aim to find the placement with the highest pressure point on a given structure and thus guarantee the highest possible output recuperated electrical charges at the electrodes [58], [59].

c) Simultaneous piezoelectric's actuation and sensing design optimization:

Some researchers have considered using both the direct and reverse effect of piezoelectric materials to ensure simultaneous good observability and controllability of a structure. Moheimani et al. [60] proposed an electrode pattern on a piezoelectric tube actuator to simultaneous sensing and actuation. Moussa et al. [61] used a topological optimization method to design a compliant microactuator that optimally integrates actuating and sensing areas in a monolithic structure. Rougeot et al. [62] introduced a three-layered piezoelectric cantilever design 
for which the upper and lower layers were used for the PEA's actuation whilst the middle layer served for the sensing of the PEA's displacement and perceived force. In [60][62] approaches, the actuation and sensing do not share the same electrodes. Therefore, the resulting structure cannot be considered to be an optimized PEA's design for charge-based SSA.

Masson et al. [63] presented an anlytical approach for the design of piezoelectric cantilever actuators which aims to improve the SSA performace for external loads estimation while minimizing the dielectric effect $\left(C_{p e a} \cdot v_{c}\right)$. The optimized piezoelectric cantilever mechanical structure they proposed was supposed to achieve a tip displacement of at least $\delta^{\text {min }}=$ $25 \mu \mathrm{m}$, a minimum blocking force of $F_{b l}^{\min }=100 \mathrm{mN}$ and withstand a maximum electric field of $E_{3}^{\max }=3 \mathrm{~V} / \mu \mathrm{m}$ (depolarizing field). The optimization problem was formulated as follows:

$$
\begin{array}{crl}
\underset{l, w, h}{\operatorname{maximize}} & F(l, w, h) & =\frac{Q_{\delta}}{F_{\text {ext }}\left(C_{\text {pea }} \cdot v_{c}\right)} \\
\text { subject to } & E_{3}\left(h_{p}\right) & \leqslant 3 \mathrm{~V} / \mu \mathrm{m}, \\
F_{b l}(l, w, h) & \geqslant 100 \mathrm{mN}, \\
\delta(l, w, h) & \geqslant 25 \mu \mathrm{m}
\end{array}
$$

where $C_{p e a}$ is the PEA's capacitance in the absence of mechanical deformation and null electric field.

The approach suggested an optimization for the external load estimation $\left(\frac{Q_{\delta}}{F_{\text {ext }}} \equiv \frac{Q_{\delta}}{\delta_{m e c}}\right)$ and did not account for the piezoelectric actuation induced charges $\left(\frac{Q_{\delta}}{\delta_{\text {pre }}}\right)$. A simplified schematic of the superposition of bending under external load and piezoelectric bendind due to the reverse piezoelectric effect is shown in figure 7 to help perceive the difference between both. Furthermore, the objective function $F(l, w, h)$ just constraints the minimum displacement and blocking force and does not aim to maximize the actuation $\left(\frac{\delta_{p r e}}{v_{c}}\right)$.

As highlithed in this subsection, earlier studies on the optimal design of piezoelectric structures aimed to optimize the sensor's sensitivity to mechanical loads and to increase the actuation. None intended to optimize both simultaneously in a SSA aspect, that is, using the same electrodes for both actuation and sensing.

More recently, Bafumba et al [64] proposed an analysis of the charge-based SSA's performances dependence on the choice of the geometry and the properties of the constituent materials in case of a piezoelectric cantilever. They presented an analytical model for this type of PEA, and defined a multiobjective function for simultaneous piezoelectric's actuation and sensing design optimization where actuation and sensing share the same electodes. Experiments were conducted to corroborate the analytical modeling and analysis. Their studies and experiments results indicated a trade-off between the PEA's actuation and sensor sensitivity resulting from the choice of the mechanical properties of its constituent materials. Increasing the PEA's sensor sensitivity will be at the cost of its actuation and vice-versa. In this case, the objective function is said to be conflicting, and there exists a (possibly infinite) number of Pareto optimal solutions. Without a piece of additional subjective preference information on either the PEA's actuation or sensor sensitivity, all Pareto optimal solutions are considered equally good.

\section{Quasi-static SSA based on the piezoelectric direct effect}

The charge-based SSA implementation relies on the linear charge-deformation relationship of PEAs and it can be enhanced through the optimization of the PEA's geometry and constituent materials choice. However, when implementing the chargebased SSA, the charges are not directly measured but rather derived from current measurement (or voltage measurement for voltage-based SSA). That is, the rate of change of strain is measured rather than the strain itself. As a result, charge- and voltage-based SSA have been essentially used for vibration control [37], [40], [65]. Ivan et al. [39] adopted the already existing antiparallel compensation scheme by Hagood et al. [40] and added an integrator amplifier to enable static and quasistatic SSA (see figure 8). In this approach, the antiparallel compensation allows the discrimination of the strain induced current $\dot{Q}_{\delta}$ from $\dot{Q}_{d}$, the current due to the dielectric effect of the PEA. The integrator amplifier makes it possible to reconstruct $Q_{\delta}$ from $\dot{Q}_{\delta}$. Moreover, a leakage resistor $R_{L}$ is included to account for the leakage current. The reconstructed strain-induced charges $Q_{\delta}$ is then used for a static or quasistatic estimation of the PEA's deformation. This approach has been implemented and has demonstrated good potential for static and quasi-static charge-based SSA [16], [38], [61].

As for previous cases of charge-based SSA, the main disadvantage of this approach is the difficulty in completely offsetting $\dot{Q}_{d}$. Indeed, even for this approach, the reference capacitor $C_{r}$ must match the PEA's capacitance $C_{p e a}$ at all times for the charge-based SSA implementation to succeed. First, $C_{r}$ and $C_{p e a}$ need to have the same relaxation time, that is, $\tau_{r}=\tau_{p e a}$. Second, since $C_{p e a}$ value changes as a function of the control voltage, external force, temperature, etc., one cannot afford to use an offsetting capacitor $C_{r}$ with a constant value. The dynamic behavior of the PEA electric impedance implies that considering only a static equivalent model in a SSA circuit will unquestionably produce errors in the measurements of the charges generated by the piezoelement deformation and consequently, errors in the estimation of the displacement (strain) and/or the applied force.

Unfortunately, no analog electrical component fits the piezoelectric dynamic impedance (see figure 5). Notwithstanding, a numerical equivalent electrical circuit can easily be shaped to tackle this issue. The advantage of numerical processing is that it allows the use of more complex electrical impedance models of PEAs. Bafumba et al. [36] proposed a way to reduce errors in the estimation of the displacement at the tip of cantilever PEAs through their equivalent numerical impedance (see figure 9). The suggested equivalent numerical impedance considers the impact of the mechanical deformation of the piezoelectric element on its electrical impedance. It permited a more rigorous cancellation of $\dot{Q}_{d}$ and consequently a more accurate measurement of $\dot{Q}_{\delta}$, the current that reflects the PEA's deformation. Experiments are conducted to validate the proposed approach and demonstrate the efficiency thereof to estimate the displacement of the PEA for long duration measurement of constant step signal. 


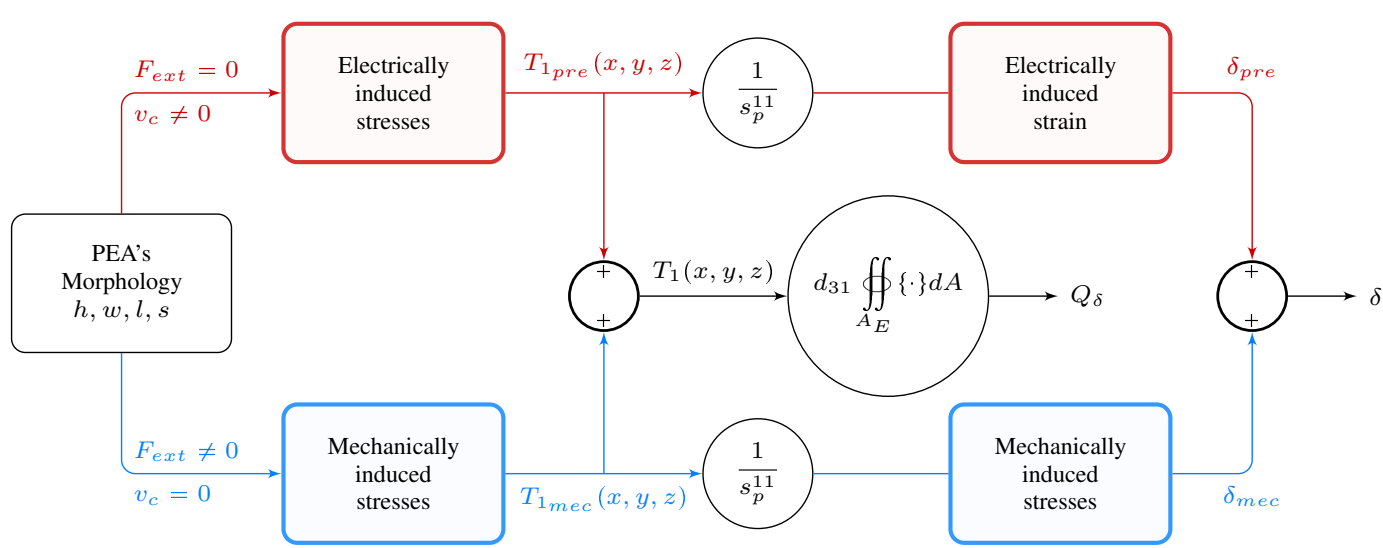

Figure 7: Schematic of the superposition of bending under external loading (-) and piezoelectric actuation (-) for an unimorph piezoelectric actuator. $h, l, s, w$ represent respectively the thickness (piezoelectric + elastic layer), the length, the compliance (piezoelectric and elastic material), and the width of the piezoelectric bender.

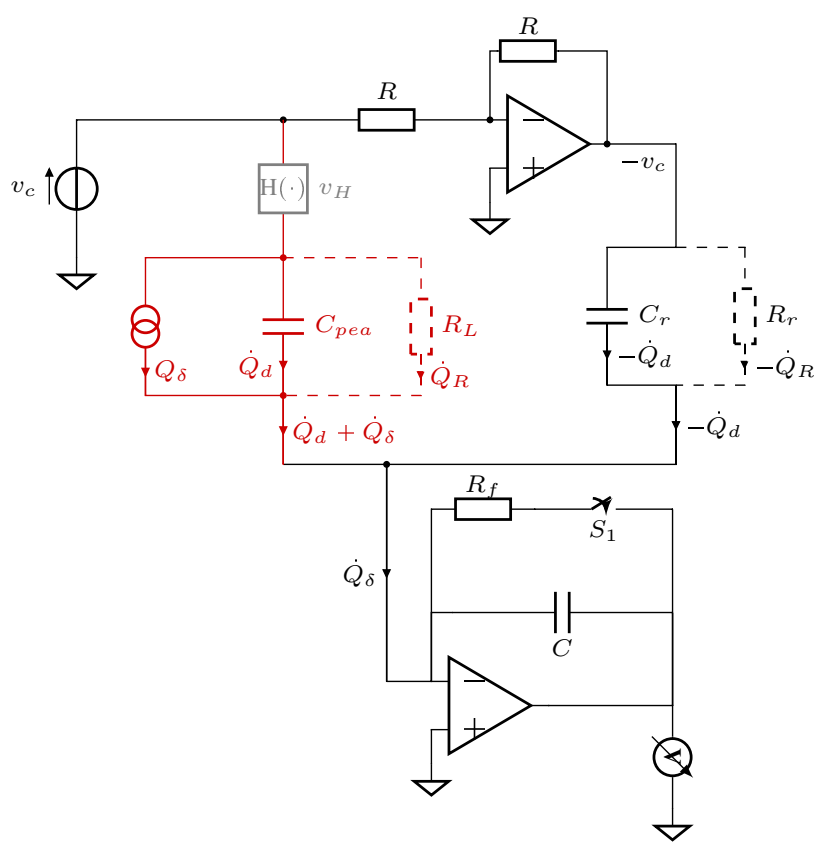

Figure 8: Electrical circuit for quasi-static charge-based SSA [39]. The dashed (- - ) part is to be considered only for lower frequency nearby $\mathrm{DC}\left(R_{r}=R_{L}\right)$.

\section{SSA BASED ON THE PEA'S CHANGE OF ELECTRICAL PROPERTIES}

\section{A. Piezoelectric Effect - Dynamic electrical properties}

SSA based on the piezoelectric direct effect demands a tedious continual tuning in order to discriminate the signal that is related to the PEA's strain from the dielectric signal, the noisy environmental conductive materials or from outer metal parts such as electrical wires [22], [36]. As already mentioned, SSA based on the piezoelectric direct effect cannot provide a good estimate of the PEA's displacement if its initial state is unknown. Whereas one already knows that applying an electric field and/or an external load induces a deformation in the PEA, recent works have shown that the PEA's impedance is also

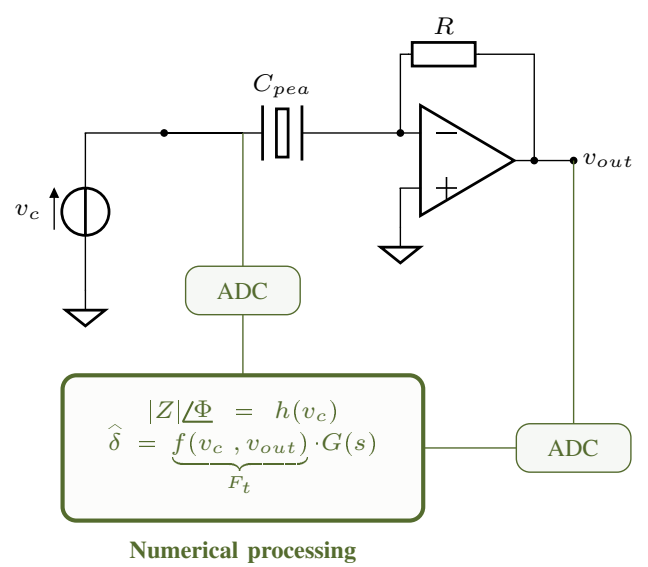

Figure 9: Simplified block diagram of the charge-based SSA considering the dynamic impedance of a PEA. $G(s)$ is the equivalent mechanical system that reliably reproduces the mechanical behavior of the PEA when subjected to $v_{c}$ and $F_{t}=f\left(v_{c}, v_{\text {out }}\right)$ is the transduced force resulting from the application of $v_{c} . F_{t}$ expresses the normal force responsible for the PEA expansion or contraction in the absence of any external mechanical stress. $h\left(v_{c}\right)$ expresses the relationship between the input voltage $v_{c}$ and the impedance magnitude change $|Z|$. ADC stand for Analog-to-Digital Converter.

affected by electric field application [36] and external loads [43]. Therefore, instead of voltage/charge-based SSA, many researchers suggested using the change of PEA's electrical properties as a means to estimate its deformation and perceived external force. Kawamata et al [66] was the first to use the linear relationship between the permittivity change and the PEA's displacement for SSA. The permittivity feedback control based on this linear relationship has shown its effectiveness for high-precision positioning [67], [68]. Since then, not only the changing relative permittivity [14], but also capacitance [69], or capacitance and resistance at the same time [43] have been used to estimate the PEA's displacement and/or perceived force. 
For an online implementation of the SSA based on the PEA's change of electrical properties, that is, an online estimation of the PEA's generated displacement and the force applied thereto based on its impedance variation, one requires a real-time impedance variation measurement scheme.

\section{B. Real-time PEA's electric properties measurement}

SSA based on the PEA's change of electrical properties relates to piezoelectric devices used for simultaneous control and displacement sensing based on the impedance variation thereof. Since the impedance variation of the PEA is a consequence of its deformation, the SSA discussed here uses a real-time measurement of the PEA's impedance to reconstruct its deformation. This is made possible thanks to a low-amplitude/high-frequency excitation signal $v_{d}$ that is added to a control signal $v_{c}$ which actuate the PEA (see figure 10). The excitation signal, also called detection signal, does not generate any deformation and only serves to evaluate the PEA's impedance variation. The control input signal $v_{c}$ is the sole responsible for the PEA's deformation and is generated according to the desired trajectory.

To successfully estimate the displacement of the PEA from the measurement of the variation of its impedance, two elements are necessary:

(1) A proper separation of the control and impedance detection signals enabling on to control the PEA and derive precise PEA's deformation information;

(2) The design of the estimator that uses the detection signal to estimate the PEA's generated displacement.

Huang et al. [70] patented a bridge circuit for both signals separation (control and impedance detection signals) and the estimation of the PEA's generated displacement. The proposed bridge circuit is connected to a piezoelectric element, and to a voltage-controlled element. The voltage-controlled element (capacitor and resistor in series) is supposed to mimic the electrical response parameters of the piezoelectric element and match at all time the current flowing out of the PEA (identified as 84 in figure 11). The current flowing out of the PEA reflects the dynamic impedance thereof. The difference between the actuator arm and the reference arm is used to adjust the voltage-controlled element and to indicate the deformation of the PEA. In addition, the system has a compensating circuit connected to the bridge circuit and the driving stage for deriving a compensation signal. The compensation signal is added to $v_{c}$ and $v_{d}$ in order to provide a damping correction, a stiffening correction and an inertial conditioning correction based on the signal received from the low-frequency difference amplifier.

Whereas SSA is supposed to allow a reduction in cost and space occupation, the proposed method by Huang et al. [70] is more likely to be bulkier than the position sensor it is supposed to replace due to the important number of elements used for its implementation. Furthermore, the control and detection voltage, $v_{c}$ and $v_{d}$ respectively, to be applied to the system are chosen so that they do not cause saturation or nonlinear behavior of the PEA. This limits the control voltage to few tens of volts and does not exploit the full range of the piezoelectric element actuation.
Instead of a single electrical circuit to obtain the impedance detection signal and estimate the PEA's generated displacement, Saigusa et al. [14] opted for a two stages approach. First, a differential current measurement associated to a lock-in amplifier to capture the PEA's impedance variation of a bimorph actuator. Thence the estimator, a third order polynomial of the measured current amplitude, is used to estimate the PEA's generated displacement. Whereas this method is supposed not to be restricted as to the input voltage to be applied for actuation and detection, considering the relative permittivity of the PEA as the unique parameter to estimate the generated displacement thereof is only accurate for small deformation of the piezoelectric element and a limited range of the detection signal frequency. Indeed, the authors made two hypotheses for their approach to hold true: (1) The piezoelectric element is modeled as a capacitor, (2) The permittivity is proportional to the current amplitude because the dimensions of the PEA, such as the thickness and electrode size, can be considered to be constant. For the first hypothesis, the equivalent electric model of a piezoelectric element is frequency dependent, ranging from a capacitor to an inductor. Therefore, modeling the PEA as a capacitor is only accurate for a fixed frequency range of the detection signal. For the second hypothesis, only small deformations of the PEA in the $x$-direction may have us neglect the deformation in the other directions. Nevertheless, a more valid approach would suggest linking the current amplitude to the capacitance variation of the piezoelectric element. Thence, the capacitance variation would be used to estimate the PEA's generated displacement [69], [71], [72]. Furthermore, a lock-in amplifier is an expensive and cumbersome instrument that hindrances the cost and space reduction that SSA is supposed to bring.

Seethaler et al. [73] patented an apparatus and method for in-situ impedance measurement of a PEA located in a fuel injector of a combustion chamber of an internal combustion. They suggested the use of a voltage sensor, a current sensor and a computer for a real-time computation of the PEA's impedance variation. The proposed method uses a Discrete Fourier Transform (DFT) to extract the voltage and current coefficient from the discrete frequency-domain and compute the PEA's impedance as a ratio of the voltage and current at the detection frequency $f_{d}$, that is:

$$
Z=\frac{v_{d}}{I_{d}}=R-\frac{j}{2 \pi f_{d} C_{p e a}}
$$

where $v_{d}$ and $I_{d}$ represent the digitized detection voltage and current respectively.

Referring to Eq. 7, the impedance $Z$ modeling the PEA includes a resistor $R$ in series with the PEA's capacitance $C_{\text {pea }}$. The conducted experiment to derive this model indicated a phase shift of $60^{\circ}$ between $v_{d}$ and $I_{d}$. Although other configurations can be used to produce the same phase shift, the authors opted for a resistor and capacitor in series. The proposed in-situ impedance measurement was used by Mansour et al. [43] in a third order polynomial fit to estimate the PEA's generated displacement and the force applied thereto. To the best of our knowledge, the apparatus and method suggested 


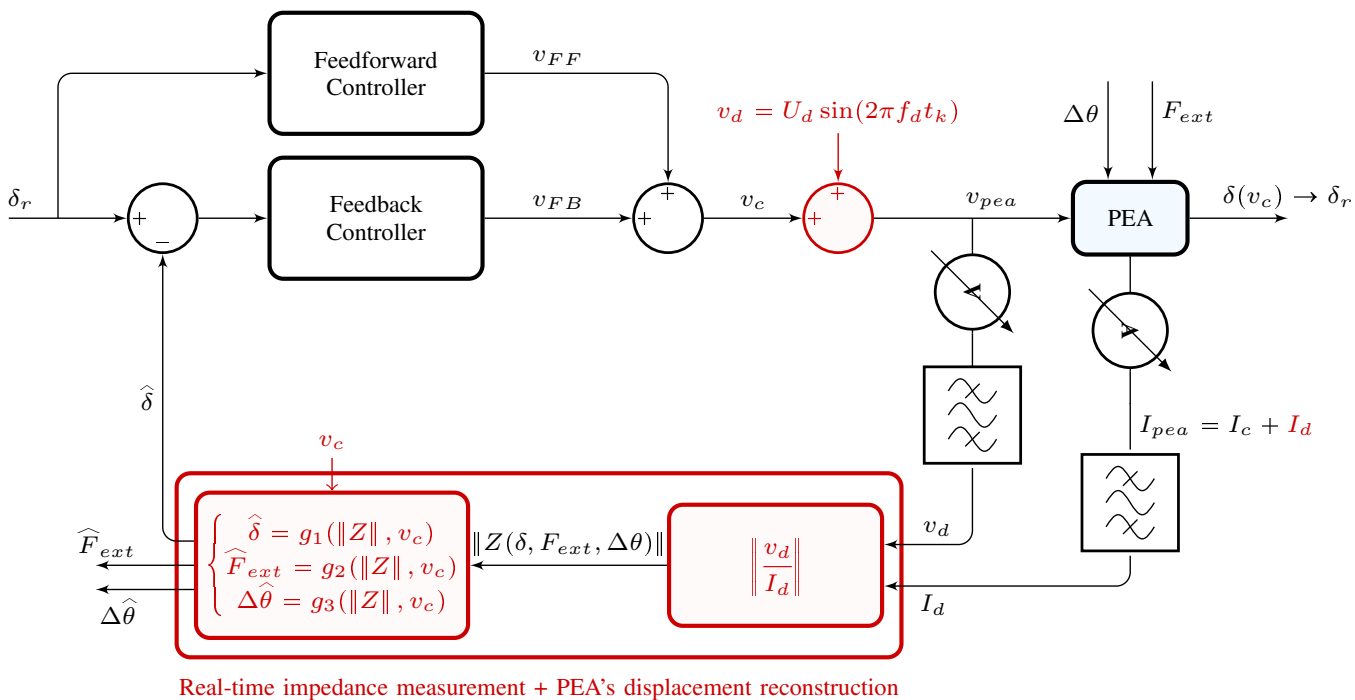

Figure 10: Block diagram : Simultaneous control and displacement measurement of a PEA using SSA based on the PEA's change of electrical properties. $F_{e x t}$ is the applied external force, $\Delta \theta$ it the temperature variation and $\left.g_{i}(\cdot)\right|_{i=1,2,3}$ represent the estimator used to reconstruct the PEA's displacement $\hat{\delta}$, perceived force $\hat{F}_{\text {ext }}$ and surrounding temperature variation $\Delta \hat{\theta}$ from the real-time calculation of its impedance magnitude change $|Z|$ and the applied input voltage $v_{c}$.

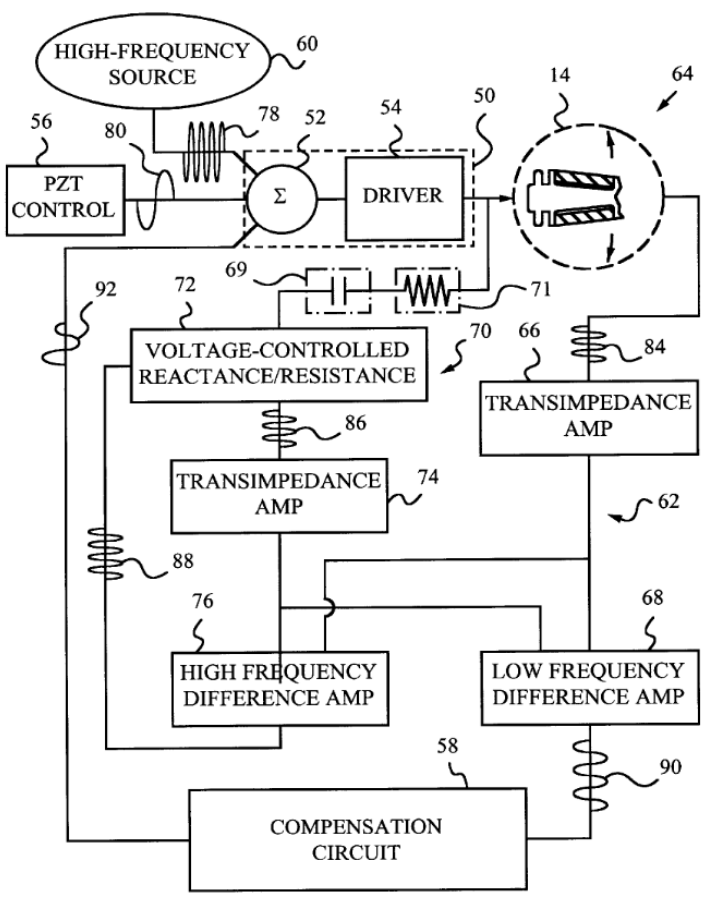

Figure 11: Block diagram of the Self-Sensing bridge circuit for controlling and sensing the response of PEAs [70]. $50-$ Driving input stage, $80-v_{c}, 78-v_{d}, 14-\mathrm{PEA}, 70$ - reference arm of the bridge, 62 - actuator arm of the bridge circuit, 84 - current flowing out of the PEA. by [73] constitute the most packageable implementation for SSA based on PEA's change of electric properties.

SSA based on piezoelectric direct effect uses charge measurement to infer the PEA's generated displacement. This technique faces a challenge with charge drift due to offset currents. To eliminate the charge drift and allow static and quasi-static applications, Ivan et al. [39] presented a simplified current integrator circuit (modified charge amplifier). However, this requires additional circuitry. For SSA based on the PEA's change of electrical properties, like the one introduced in [72], the PEA's generated displacement is obtained from a real-time measurment of the effective capacitance of the PEA. However, this technique suffers from considerable noise and hence can only be used for slow operations. Islam et al. [44] developed a hybrid position observer (HPO) that fused the capacitancebased SSA estimate with the charge-based SSA estimate into a single high quality position estimate (see figure 12). The first position estimate uses the well documented linear relationship between charge and position [1], [39], [74], [75] which provides a highly dynamic position signal which unfortunately suffers from drift due to charge leakage. The second position estimate is obtained from the relationship between effective actuator capacitance and position [69], [72]. Due to high frequency noise, this second relationship can only provide low-frequency position estimates. However, since drift in the first estimate is a low frequency phenomenon, it can be eliminated with the second estimate using the HPO. Once a reliable position signal is achieved, it can be used in a traditional feedback controller replacing the dedicated position sensor.

\section{SimUltaneOUS FORCE AND POSITION ESTIMATION THROUGH SSA}

Nanopositioners could perform a variety of precision machining operations [76], [77] and they might be configured 


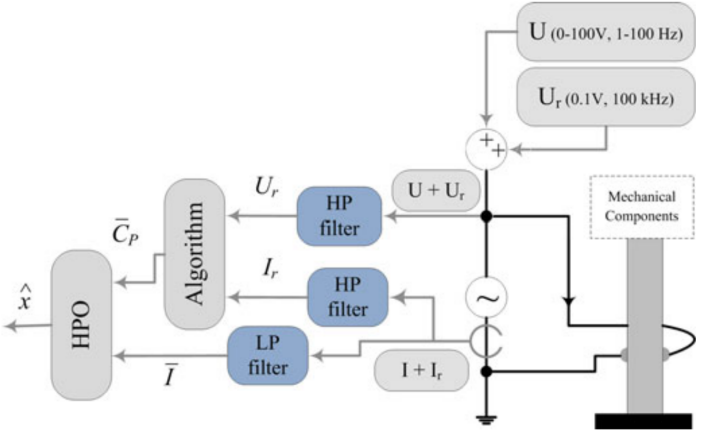

Figure 12: Flowchart of the Hybrid SSA position observer [44]. $\bar{C}_{p}$ is the PEA effective capacitance and $\hat{x}$ is the PEA's position estimate using the hybrid SSA observer. $U \equiv v_{c}$ and $U_{r} \equiv v_{d}$.

to assemble micrometer-sized parts [78]-[80]. However, it is worth noting that microscopic objects are generally fragile (objects of small dimensions that are often made of special materials) so that the forces exerted during their handling must be adapted and controlled. Whereas all the listed SSA techniques in previous sections have often been used for constant force operating conditions, applications such as microgrippers [81], [82] exhibit both varying displacement and force. Due to the additional constraint of the handling force of microobjects, some researchers have focused on the development of SSA's techniques that allow the simultaneous estimation of displacement and force. These techniques exploit either the piezoelectric direct effect or the PEA's change of electrical properties.

Two measurements are required to simultaneously estimate displacement and force. The first attempts for simultaneous displacement and force estimation were extensions of chargebased SSA techniques. Badel et al. [83] proposed a model that computes the generated force and the elongation of the PEA from the measurements of the produced electrical charges and input voltage applied to the PEA. This model included a dedicated hysteresis operator that allowed the hysteretic properties of the PEA to be taken into account. The detection model combined with a PID controller has been used successfully, and experiments conducted to show the feasibility of SSA for force control of a PEA. An identical approach was used by McPherson et al. [45] to estimate the force and displacement at the tip of a piezoelectric-based driven magnetic resonance imaging (MRI)-compatible tweezer and by Mansour et al. [84], [85] for simultaneous displacement and force estimation of piezoelectric stack actuators. Ivan et al. [86], like in [45], [83], [84], used the measured electrical charges, the input voltage, an hysteresis operator and added an ARMAX model to account for the creep effect and provide a more reliable estimate of the steady-state PEA's generated displacement and force applied thereto.

Rakotondrabe et al. [16] extended the proposed scheme in [86] to include the SSA scheme for dynamic displacement estimation suggested in [87], [88] and thus allow the estimation of the PEA's generated displacement in both steady-state and dynamics while the force estimation remained in the steady- state. In the proposed approach, the estimated displacement was used as a feedback signal whereas the estimated force was only being displayed. In order to estimate the PEA's generated displacement, the force applied thereto and state, all in a full way (steady-state and dynamics, i.e. low and highfrequency), Rakotondrabe et al. [89] introduced an Unknown Input Observer (UIO) in the SSA approach proposed in [87]. The main advantages of the latter over previous SSA approaches are 1) the possibility of feedback control for the displacement and for the force, 2) and the possibility to use modern control such as state-feedback.

The SSA technique introduced in [86] was destined for the simultaneous estimation of the PEA's generated displacement and force applied thereto for steady-state. One drawback of this method is having relatively large transient errors, which makes it hard to be used in fast applications. Before its extension to a full (i.e. low and high-frequency) displacement, force and state estimation [89], Mansour et al. [90] proposed an alternative approach for simultaneous estimation of the displacement and force for both the transient and steady-state. Rather than using measurements of produced charges and the input voltage, this method utilizes measurements of the produced charges and effective capacitance. This approach eliminates the need for a mathematically complex inverse hysteresis models and is supposed to be largely rate independent as well as robust against the creep.

More recently, Mansour et al. [43] proposed a technique for real-time detection of the variation of the capacitance and resistance of the PEA at a resonance frequency and a third order polynomial fit for the simultaneous estimation of the displacement and force. Conducted experiments with this technique have provided estimates of the displacement and force equivalent or better than previously reported displacement-force SSA techniques that use charge and voltage measurements. However, the performances for dynamic operations of the technique proposed in [43] yet need to be investigated.

\section{CONClusion}

Nanopositioning tasks, which are the basis of microassembly and micromanipulation applications, require very high precision while being limited by space and cost factors that hinder the insertion of external position and force sensors to enable quality and robust servo control. SSA techniques provide a valuable alternative to the traditional means for the acquisition of position and force observations where mounting a dedicated hardware sensor is not feasible or unsuitable. This paper reviewed the two SSA techniques used for precise positioning control of PEAs, namely: 1) SSA based on the piezoelectric direct effect and, 2) SSA based on the PEA's change of electrical properties. This paper also presented current and emerging SSA techniques that aim for a simultaneous displacement and force estimation to enable precision control with nanometer-scale positioning and the control of the handling force during micro-objects manipulation.

\section{ACKNOWLEDGMENT}

This work has been supported by the EIPHI Graduate school (contract "ANR-17-EURE-0002"). 


\section{REFERENCES}

[1] S. Devasia, E. Eleftheriou, and S. R. Moheimani, "A survey of control issues in nanopositioning," IEEE Transactions on Control Systems Technology, vol. 15, no. 5, pp. 802-823, 2007.

[2] G. Lee, K. You, T. Kang, K. J. Yoon, J. O. Lee, and J. K. Park, "Modeling and design of h-infinity controller for piezoelectric actuator lipca," Journal of Bionic Engineering, vol. 7, no. 2, pp. 168-174, 2010.

[3] X. Tan and J. S. Baras, "Adaptive identification and control of hysteresis in smart materials," IEEE Transactions on automatic control, vol. 50, no. 6, pp. 827-839, 2005.

[4] S. M. Salapaka and M. V. Salapaka, "Scanning probe microscopy," IEEE Control Systems, vol. 28, no. 2, pp. 65-83, 2008.

[5] G. M. Clayton, S. Tien, K. K. Leang, Q. Zou, and S. Devasia, "A review of feedforward control approaches in nanopositioning for high-speed spm," Journal of dynamic systems, measurement, and control, vol. 131, no. 6, p. 061101, 2009.

[6] W. Gao, Precision nanometrology: sensors and measuring systems for nanomanufacturing. Springer Science \& Business Media, 2010.

[7] P. P. Regtien and E. Dertien, Sensors for mechatronics. Elsevier, 2018.

[8] A. J. Fleming, "A review of nanometer resolution position sensors: Operation and performance," Sensors and Actuators A: Physical, vol. 190, pp. 106-126, 2013.

[9] C. W. De Silva, Sensors and actuators: Engineering system instrumentation. CRC Press, 2015.

[10] M. Rakotondrabe, "Bouc-wen modeling and inverse multiplicative structure to compensate hysteresis nonlinearity in piezoelectric actuators," IEEE Transactions on Automation Science and Engineering, vol. 8, no. 2 pp. 428-431, 2011.

[11] B. Mokaberi and A. A. Requicha, "Compensation of scanner creep and hysteresis for afm nanomanipulation," IEEE Transactions on Automation Science and Engineering, vol. 5, no. 2, pp. 197-206, 2008.

[12] K. Furutani, M. Urushibata, and N. Mohri, "Improvement of control method for piezoelectric actuator by combining induced charge feedback with inverse transfer function compensation," in Robotics and Automation 1998. Proceedings. 1998 IEEE International Conference on, vol. 2 , pp. 1504-1509, IEEE, 1998.

[13] A. J. Fleming and S. R. Moheimani, "A grounded-load charge amplifier for reducing hysteresis in piezoelectric tube scanners," Review of Scientific Instruments, vol. 76, no. 7, p. 073707, 2005.

[14] K. Saigusa and T. Morita, "Self-sensing control of piezoelectric positioning stage by detecting permittivity," Sensors and Actuators A: Physical, vol. 226, pp. 76-80, 2015.

[15] M. G. Ruppert and S. R. Moheimani, "High-bandwidth multimode self-sensing in bimodal atomic force microscopy," Beilstein journal of nanotechnology, vol. 7, p. 284, 2016.

[16] M. Rakotondrabe, I. A. Ivan, S. Khadraoui, P. Lutz, and N. Chaillet, "Simultaneous displacement/force self-sensing in piezoelectric actuators and applications to robust control," IEEE/ASME Transactions on Mechatronics, vol. 20, no. 2, pp. 519-531, 2015.

[17] O. A. J. Mohammad Al Janaideh, Micky Rakotondrabe, "Further results on hysteresis compensation of smart micro-positioning systems with the inverse prandtl-ishlinskii compensator," Transactions on Control Systems Technology, vol. 24, no. 2, pp. 428-439, 2015.

[18] G. Clayton, S. Tien, A. Fleming, S. Moheimani, and S. Devasia, "Inversefeedforward of charge-controlled piezopositioners," Mechatronics, vol. 18, no. 5-6, pp. 273-281, 2008.

[19] D. J. Inman, "Control/structure interaction: effects of actuator dynamics," Mechanics and Control of Large Structures, pp. 507-533, 1990.

[20] G. E. Simmers Jr, H. A. Sodano, G. Park, and D. J. Inman, "Thermal protection for a self-sensing piezoelectric control system," Smart Materials and Structures, vol. 16, no. 6, p. 2492, 2007.

[21] J. J. Dosch, D. J. Inman, and E. Garcia, "A self-sensing piezoelectric actuator for collocated control," Journal of Intelligent material systems and Structures, vol. 3, no. 1, pp. 166-185, 1992.

[22] S. R. Moheimani, "A survey of recent innovations in vibration damping and control using shunted piezoelectric transducers," IEEE transactions on control systems technology, vol. 11, no. 4, pp. 482-494, 2003.

[23] K. Makihara, J. Onoda, and K. Minesugi, "A self-sensing method for switching vibration suppression with a piezoelectric actuator," Smart Materials and Structures, vol. 16, no. 2, p. 455, 2007.

[24] S. R. Moheimani, "Invited review article: Accurate and fast nanopositioning with piezoelectric tube scanners: Emerging trends and future challenges," Review of Scientific Instruments, vol. 79, no. 7, p. 071101, 2008.
[25] C. K. Pang, G. Guo, B. M. Chen, and T. H. Lee, "Self-sensing actuation for nanopositioning and active-mode damping in dual-stage hdds," IEEE/ASME Transactions on Mechatronics, vol. 11, no. 3, pp. 328$338,2006$.

[26] A. J. Fleming and S. R. Moheimani, "Sensorless vibration suppression and scan compensation for piezoelectric tube nanopositioners," IEEE Transactions on Control Systems Technology, vol. 14, no. 1, pp. 33-44, 2006.

[27] M. Goldfarb and N. Celanovic, "A lumped parameter electromechanical model for describing the nonlinear behavior of piezoelectric actuators," Journal of dynamic systems, measurement, and control, vol. 119, no. 3, pp. 478-485, 1997.

[28] H. Adriaens, W. L. De Koning, and R. Banning, "Modeling piezoelectric actuators," IEEE/ASME transactions on mechatronics, vol. 5, no. 4, pp. 331-341, 2000.

[29] Y. Gao, D. Zhang, and C. W. Yu, "Dynamic modeling of a novel workpiece table for active surface grinding control," International Journal of Machine Tools and Manufacture, vol. 41, no. 4, pp. 609-624, 2001.

[30] M. Quant, H. Elizalde, A. Flores, R. Ramírez, P. Orta, and G. Song, "A comprehensive model for piezoceramic actuators: modelling, validation and application," Smart Materials and Structures, vol. 18, no. 12, p. 125011, 2009.

[31] H. Georgiou and R. B. Mrad, "Dynamic electromechanical drift model for pzt," Mechatronics, vol. 18, no. 2, pp. 81-89, 2008.

[32] K. Seki and M. Iwasaki, "Application of self-sensing technique for position control considering vibration suppression in piezo-driven stage," in Mechatronics (ICM), 2015 IEEE International Conference on, pp. 278289, IEEE, 2015

[33] T. Takigami, K. Oshima, and Y. Hayakawa, "Application of self-sensing actuator to control of a cantilever beam," in American Control Conference, 1997. Proceedings of the 1997, vol. 3, pp. 1867-1872, IEEE, 1997.

[34] P. Albertelli, S. Elmas, M. R. Jackson, G. Bianchi, R. M. Parkin, and M. Monno, "Active spindle system for a rotary planing machine," The International Journal of Advanced Manufacturing Technology, vol. 63, no. 9-12, pp. 1021-1034, 2012.

[35] Y. He, X. Chen, Z. Liu, and Y. Qin, "Piezoelectric self-sensing actuator for active vibration control of motorized spindle based on adaptive signal separation," Smart Materials and Structures, vol. 27, no. 6, p. 065011, 2018.

[36] J. B. Liseli, J. Agnus, P. Lutz, and M. Rakotondrabe, "Self-sensing method considering the dynamic impedance of piezoelectric based actuators for ultralow frequency," IEEE Robotics and Automation Letters, vol. 3, no. 2, pp. 1049-1055, 2018.

[37] M. Sasaki and S. Ito, "Vibration control of a micro-actuator for the hard disk drive using self-sensing actuation," in Control, Automation and Systems, 2008. ICCAS 2008. International Conference on, pp. 2855-2860, IEEE, 2008.

[38] I. A. Ivan, O. Aljanaideh, J. Agnus, P. Lutz, and M. Rakotondrabe, "Quasistatic displacement self-sensing measurement for a 2-dof piezoelectric cantilevered actuator," IEEE Transactions on Industrial Electronics, vol. 64, no. 8, pp. 6330-6337, 2017.

[39] I. A. Ivan, M. Rakotondrabe, P. Lutz, and N. Chaillet, "Quasistatic displacement self-sensing method for cantilevered piezoelectric actuators,' Review of Scientific instruments, vol. 80, no. 6, p. 065102, 2009.

[40] N. W. Hagood and E. H. Anderson, "Simultaneous sensing and actuation using piezoelectric materials," in Active and Adaptive Optical Components, vol. 1543, pp. 409-422, International Society for Optics and Photonics, 1992.

[41] C. H. Park, "On the circuit model of piezoceramics," Journal of Intelligent Material Systems and Structures, vol. 12, no. 7, pp. 515-522, 2001.

[42] M. Guan and W.-H. Liao, "Studies on the circuit models of piezoelectric ceramics," in Information Acquisition, 2004. Proceedings. International Conference on, pp. 26-31, IEEE, 2004.

[43] S. Z. Mansour and R. Seethaler, "Simultaneous quasi-static displacement and force self-sensing of piezoelectric actuators by detecting impedance," Sensors and Actuators A: Physical, vol. 274, pp. 272-277, 2018.

[44] M. N. Islam and R. J. Seethaler, "Sensorless position control for piezoelectric actuators using a hybrid position observer," IEEE/ASME Transactions On Mechatronics, vol. 19, no. 2, pp. 667-675, 2014.

[45] T. McPherson and J. Ueda, "A force and displacement self-sensing piezoelectric mri-compatible tweezer end effector with an on-site calibration procedure," IEEE/ASME Transactions on Mechatronics, vol. 19, no. 2, pp. 755-764, 2014.

[46] M. Lebedev and J. Akedo, "What thickness of the piezoelectric layer with high breakdown voltage is required for the microactuator?," Japanese journal of applied physics, vol. 41, no. 5S, p. 3344, 2002. 
[47] K. Hu and H. Li, "Multi-parameter optimization of piezoelectric actuators for multi-mode active vibration control of cylindrical shells," Journal of Sound and Vibration, vol. 426, pp. 166-185, 2018.

[48] S. Khadraoui, M. Rakotondrabe, and P. Lutz, "Optimal design of piezoelectric cantilevered actuators with guaranteed performances by using interval techniques," IEEE/ASME Transactions on Mechatronics, vol. 19, no. 5, pp. 1660-1668, 2014.

[49] S. Canfield and M. Frecker, "Topology optimization of compliant mechanical amplifiers for piezoelectric actuators," Structural and Multidisciplinary Optimization, vol. 20, no. 4, pp. 269-279, 2000.

[50] F. Wein, M. Kaltenbacher, E. Bänsch, G. Leugering, and F. Schury, "Topology optimization of a piezoelectric-mechanical actuator with singleand multiple-frequency excitation," International Journal of Applied Electromagnetics and Mechanics, vol. 30, no. 3, 4, pp. 201-221, 2009.

[51] A. Donoso and O. Sigmund, "Topology optimization of piezo modal transducers with null-polarity phases," Structural and Multidisciplinary Optimization, vol. 53, no. 2, pp. 193-203, 2016.

[52] M. Kögl and E. C. Silva, "Topology optimization of smart structures: design of piezoelectric plate and shell actuators," Smart materials and Structures, vol. 14, no. 2, p. 387, 2005.

[53] J. F. Gonçalves, D. M. De Leon, and E. A. Perondi, "Simultaneous optimization of piezoelectric actuator topology and polarization," Structural and Multidisciplinary Optimization, vol. 58, no. 3, pp. 1139-1154, 2018

[54] D. Ruiz, J. Bellido, and A. Donoso, "Design of piezoelectric modal filters by simultaneously optimizing the structure layout and the electrode profile," Structural and Multidisciplinary Optimization, vol. 53, no. 4 pp. 715-730, 2016.

[55] M. A. Pillai, D. Ebenezer, and E. Deenadayalan, "Design and optimization of piezoelectric unimorph beams with distributed excitation," The Journal of the Acoustical Society of America, vol. 143, no. 5, pp. 2685-2696, 2018.

[56] T. Schlinquer, A. Mohand-Ousaid, and M. Rakotondrabe, "Optimal design of a unimorph piezoelectric cantilever devoted to energy harvesting to supply animal tracking devices," IFAC-PapersOnLine, vol. 50, no. 1, pp. 14600-14605, 2017.

[57] N. Chen and V. Bedekar, "Modeling, simulation and optimization of piezoelectric bimorph transducer for broadband vibration energy harvesting in multi-beam and trapezoidal approach," Journal of Materials Science Research, vol. 7, no. 2, p. 26, 2018.

[58] J. Johari and T. M. A. T. Rashid, "Optimization of piezoelectric transducer placement in shoe insole for energy harvesting," in 2017 International Conference on Electrical, Electronics and System Engineering (ICEESE), pp. 61-66, IEEE, 2017.

[59] V. Gupta, M. Sharma, and N. Thakur, "Optimization criteria for optimal placement of piezoelectric sensors and actuators on a smart structure: a technical review," Journal of Intelligent Material Systems and Structures, vol. 21, no. 12, pp. 1227-1243, 2010.

[60] S. R. Moheimani and Y. K. Yong, "A new piezoelectric tube scanner for simultaneous sensing and actuation," in American Control Conference, 2009. ACC'09., pp. 2249-2253, IEEE, 2009.

[61] R. E. K. Moussa, M. Grossard, M. Boukallel, A. Hubert, and N. Chaillet, "Modeling and control of a piezoelectric microactuator with proprioceptive sensing capabilities," Mechatronics, vol. 24, no. 6, pp. 590-604, 2014.

[62] P. Rougeot, A. Mohand-Ousaid, D. Gendreau, M. Hammouche, and M. Rakotondrabe, "Design, modeling and simulation of a three-layer piezoelectric cantilevered actuator with collocated sensor," in Sensors for Next-Generation Robotics III, vol. 9859, p. 98590F, International Society for Optics and Photonics, 2016.

[63] L. Masson, L. Xinchang, and Y. Perriard, "Design of an optimized self-sensing piezoelectric cantilever for micro-robotic applications," in International Conference on Manipulation, Automation and Robotics at Small Scales, IEEE, 2018.

[64] J. Bafumba Liseli, J. Agnus, P. Lutz, and M. Rakotondrabe, "Optimal design of piezoelectric cantilevered actuators for charge-based selfsensing applications," Sensors, vol. 19, no. 11, p. 2582, 2019.

[65] T. Rittenschober and K. Schlacher, "Observer-based self sensing actuation of piezoelastic structures for robust vibration control," Automatica, vol. 48 no. 6, pp. 1123-1131, 2012.

[66] A. Kawamata, Y. Kadota, H. Hosaka, and T. Morita, "Self-sensing piezoelectric actuator using permittivity detection," Ferroelectrics, vol. 368, no. 1, pp. 194-201, 2008.

[67] H. Ikeda and T. Morita, "High-precision positioning using a self-sensing piezoelectric actuator control with a differential detection method," Sensors and Actuators A: Physical, vol. 170, no. 1-2, pp. 147-155, 2011.

[68] Y. Ishikiriyama and T. Morita, "Improvement of self-sensing piezoelectric actuator control using permittivity change detection," Journal of Advanced
Mechanical Design, Systems, and Manufacturing, vol. 4, no. 1, pp. $143-$ 149,2010

[69] M. Islam, R. Seethaler, and D. Mumford, "Hysteresis independent on-line capacitance measurement for piezoelectric stack actuators," in Electrical and Computer Engineering (CCECE), 2011 24th Canadian Conference on, pp. 001149-001153, IEEE, 2011.

[70] F.-Y. Huang, W. I. Imaino, and J.-C. Lo, "Piezoelectric actuator for control and displacement sensing," Aug. 8 2000. US Patent 6,100,623.

[71] Y. Cho and F. Matsuno, "Dynamic measuring method of capacitance variation of piezoelectric ceramics with alternating electric field," Japanese journal of applied physics, vol. 31, no. 11R, p. 3627, 1992.

[72] M. N. Islam and R. J. Seethaler, "Identification of constitutive parameters for piezo stack actuators based on online capacitance measurements," in ASME 2011 International Design Engineering Technical Conferences and Computers and Information in Engineering Conference, pp. 457-463, American Society of Mechanical Engineers, 2011.

[73] M. I. Rudolph SEETHALER, "Apparatus and method for in situ impedance measurement of a piezoelectric actuator," Aug. 82011 WO2012149649A1.

[74] M. Goldfarb and N. Celanovic, "Modeling piezoelectric stack actuators for control of micromanipulation," IEEE Control Systems Magazine, vol. 17 , no. 3, pp. 69-79, 1997.

[75] A. Fleming and S. Moheimani, "Improved current and charge amplifiers for driving piezoelectric loads, and issues in signal processing design for synthesis of shunt damping circuits," Journal of Intelligent Material Systems and Structures, vol. 15, no. 2, pp. 77-92, 2004.

[76] P. Gao, H. Tan, and Z. Yuan, "The design and characterization of a piezo-driven ultra-precision stepping positioner," Measurement Science and Technology, vol. 11, no. 2, p. N15, 2000.

[77] S.-S. Ku, U. Pinsopon, S. Cetinkunt, and S.-i. Nakajima, "Design, fabrication and real-time neural network control of a three degrees of freedom nano-positioner," in Advanced Intelligent Mechatronics' 97. Final Program and Abstracts., IEEE/ASME International Conference on, p. 99, IEEE, 1997.

[78] P. Gao and S.-M. Swei, "A six-degree-of-freedom micro-manipulator based on piezoelectric translators," Nanotechnology, vol. 10, no. 4, p. 447, 1999.

[79] K. S. Jung and Y. S. Baek, "Contact-free moving-magnet type of micropositioner with optimized specification," IEEE transactions on magnetics, vol. 38, no. 3, pp. 1539-1548, 2002.

[80] Y. Sun, D. Piyabongkarn, A. Sezen, B. Nelson, and R. Rajamani, "A high-aspect-ratio two-axis electrostatic microactuator with extended travel range," Sensors and Actuators A: Physical, vol. 102, no. 1-2, pp. 49-60, 2002.

[81] S. Yang and Q. Xu, "A review on actuation and sensing techniques for mems-based microgrippers," Journal of Micro-Bio Robotics, vol. 13, no. 1-4, pp. 1-14, 2017.

[82] Y. Wei and Q. Xu, "An overview of micro-force sensing techniques," Sensors and Actuators A: Physical, vol. 234, pp. 359-374, 2015.

[83] A. Badel, J. Qiu, and T. Nakano, "Self-sensing force control of a piezoelectric actuator," IEEE transactions on ultrasonics, ferroelectrics, and frequency control, vol. 55, no. 12, pp. 2571-2581, 2008.

[84] S. Z. Mansour and R. J. Seethaler, "Simultaneous displacement and force estimation of piezoelectric stack actuators using charge and voltage measurements," IEEE/ASME Transactions on Mechatronics, vol. 22, no. 6, pp. 2619-2624, 2017.

[85] S. Z. Mansour and R. Seethaler, "Displacement and force self-sensing technique for piezoelectric actuators using a nonlinear constitutive model," IEEE Transactions on Industrial Electronics, 2019.

[86] I. A. Ivan, M. Rakotondrabe, P. Lutz, and N. Chaillet, "Current integration force and displacement self-sensing method for cantilevered piezoelectric actuators," Review of Scientific Instruments, vol. 80, no. 12, p. 126103 , 2009.

[87] M. Rakotondrabe, I. A. Ivan, S. Khadraoui, C. Clévy, P. Lutz, and N. Chaillet, "Dynamic displacement self-sensing and robust control of cantilever piezoelectric actuators dedicated for microassembly," in 2010 IEEE/ASME International Conference on Advanced Intelligent Mechatronics, pp. 557-562, IEEE, 2010.

[88] O. Aljanaideh and M. Rakotondrabe, "Observer and robust $h_{\infty}$ control of a 2-dof piezoelectric actuator equipped with self-measurement," IEEE Robotics and Automation Letters, vol. 3, no. 2, pp. 1080-1087, 2018.

[89] M. Rakotondrabe, "Combining self-sensing with an unkown-inputobserver to estimate the displacement, the force and the state in piezoelectric cantilevered actuators," in 2013 American Control Conference, pp. 4516-4523, IEEE, 2013.

[90] S. Z. Mansour and R. J. Seethaler, "On the feasibility of using measurements of charge and effective capacitance for simultaneous 
position and force self-sensing of piezoelectric actuators," in 2016 IEEE International Conference on Advanced Intelligent Mechatronics (AIM), pp. 448-451, IEEE, 2016.

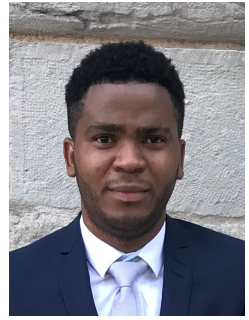

BAFUMBA LISELI Joël received an engineering degree in mechatronics from the University of São Paulo at São Carlos School of engineering in 2014, a Master of applied science degree in Robotics and Advanced Systems from Pierre and Marie Curie University in 2016 and a Ph.D degree in Automatic Control and Computer Sciences from the University of Burgundy - Franche-Comté in 2019. His main field of research is microrobotics and its applications for the design and control of high-speed motion control systems with a special emphasis on piezoelectric

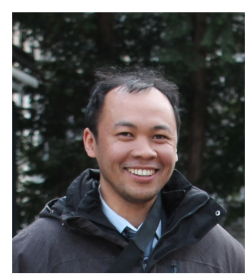

RAKOTONDRABE Micky (S'05-M'07) received the HDR degree in control systems from the Université de Franche-Comté, Besançon France, in 2014 He has been an Assistant Professor in 2006-2007 and then Associate Professor in 2007-August 2019 with the Université de Franche-Comté since 2007 with research affiliation at the FEMTO Institute, France. Since Sept 2019, he is Full Professor at the National School of Engineering (ENIT), a campus of the Toulouse INP, in Tarbes France.

Dr. Rakotondrabe is or was a member of the IEEE/RAS Technical Committee (TC) on Micro/Nano Robotics and Automation and of the IFAC TC on Mechatronics. He received several recognition prizes. In 2016, he was a recipient of the Big-On-Small Award during the IEEE MARSS International Conference. This award is to recognize a young professional $(<40 \mathrm{yo})$ with excellent performance and international visibility in the topics of mechatronics and automation for manipulation at small scales. In 2018, he is nominee for Distinguished Lecturer of Micro/Nano Robotics \& Automation at the IEEE/RAS Society. He is or was an Associate Editor or a Guest Editor in prestigious journals related to Robotics, Automation and Mechatronics (IEEE/ASME Transactions on Mechatronics, IEEE Robotics and Automation Letters, IEEE Transactions on Electronics, IFAC Mechatronics, MDPI Actuators)

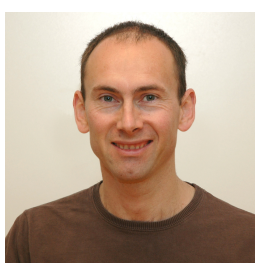

AGNUS Joël received the Master of Science in Electrical Engineering in 1994 and the Ph.D degree in Automatic Control and Computer Sciences from the University of Besançon, France, in 2003. He is a research engineer at ENSMM engineering school and FEMTO-ST / AS2M department. He is involved in microrobotics field, and more particular concerning microgrippers, piezoelectric material and piezoresistive force sensors within micromanipulation domain and for surface characterization applications.

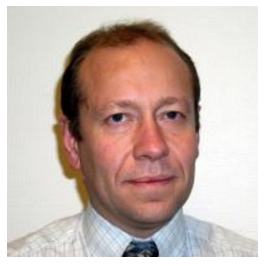

LUTZ Philippe joined the University of FrancheComté, Besancon, as Professor in 2002. Since January 2017, he is the director of the Automatic Control Research department (AS2M) of the Research Institute FEMTO-ST. He was the head of the research group "Automated Systems for Micromanipulation and Micro-assembly" of the AS2M department of FEMTO-ST Institute from 2005 to 2011. He was the Director of the PhD graduate school of Engineering science and Microsystems with more than $400 \mathrm{PhD}$ students from 2011 to February 2017, and he is currently the head of the Doctoral College of University of Bourgogne FrancheComté. His research activities at FEMTO-ST are focused on the design and control of micro-nano systems, microgrippers, micro-nano robots and micronano manipulation, assembly stations, and Optimal design and control of piezoelectrically actuated compliant structures. P. Lutz received several awards of IEEE, authored over 100 refereed publications ( 55 in high standard journals), serves as associate editor for the IEEE Transaction on Automation Science and Engineering and as Technical Editor for the IEEE/ASME Transactions on Mechatronic, is member of several steering committees and is member of the IEEE Robotics and Automation Society (RAS) Committee on Micro-Nano Robotics. 